\title{
Wireless Implantable Blood Pressure Sensing Microsystem Design for Monitoring of Small Laboratory Animals
}

\author{
Peng Cong*, Wen H. Ko and Darrin J. Young \\ 10900 Euclid Avenue, Department of Electrical Engineering and Computer Science \\ Case Western Reserve University, Cleveland, Ohio, U.S.A, 44106
}

(Received August 15, 2008; accepted November 18, 2008)

Key words: microsystem, blood pressure monitoring, RF powering, batteryless implant system, biomedical device, implantable technology, biomedical implant, MEMS pressure sensor, lowpower implant system design

A design of a novel wireless implantable blood pressure sensing microsystem for advanced biological research is presented. The system employs a miniature instrumented elastic cuff, wrapped around a blood vessel, for small laboratory animal real-time blood pressure monitoring. The elastic cuff is made of biocompatible soft silicone material by a molding process and is filled by insulating silicone oil with an immersed MEMS capacitive pressure sensor interfaced with low-power integrated electronic system. This technique avoids vessel penetration and substantially minimizes vessel restriction due to the soft cuff elasticity, and is thus attractive for long-term implant. The MEMS pressure sensor detects the coupled blood pressure waveform caused by the vessel expansion and contraction, followed by amplification, 11-bit digitization, and wireless FSK data transmission to an external receiver. The integrated electronics are designed with capability of receiving RF power from an external power source and converting the RF signal to a stable $2 \mathrm{~V}$ DC supply in an adaptive manner to power the overall implant system, thus enabling the realization of stand-alone batteryless implant microsystem. The electronics are fabricated in a $1.5 \mu \mathrm{m}$ CMOS process and occupy an area of $2 \mathrm{~mm}$ $\times 2 \mathrm{~mm}$. The prototype monitoring cuff is wrapped around the right carotid artery of a laboratory rat to measure real-time blood pressure waveform. The measured in vivo blood waveform is compared with a reference waveform recorded simultaneously using a commercial catheter-tip transducer inserted into the left carotid artery. The two measured waveforms are closely matched with a constant scaling factor. The ASIC is interfaced with a 5-mm-diameter RF powering coil with four miniature surface-mounted components (one inductor and three capacitors) over a thin flexible substrate by bond wires, followed by silicone coating and packaging with the prototype blood pressure monitoring cuff. The overall system achieves a measured average sensitivity of 7 LSB/ mmHg, a nonlinearity less than $2.5 \%$ of full scale, and a hysteresis less than $1 \%$ of full scale. From noise characterization, a blood vessel pressure change sensing resolution *Corresponding author: e-mail: pxc59@case.edu 
of $1 \mathrm{mmHg}$ can be expected. The system weighs $330 \mathrm{mg}$, representing an order of magnitude mass reduction compared with state-of-the-art commercial technology.

\section{Introduction}

DNA sequencing of small laboratory animals together with in vivo real-time biological information, such as blood pressure, core body temperature, activity, and biopotential signals, is ultimately crucial for advanced biomedical and system biology research to identify genetic variation susceptibility to diseases, and to potentially develop new treatment methods. ${ }^{(1)}$ A miniature, light-weight, long-term, reliable biosensing implant system with wireless data telemetry and adaptive RF powering capabilities is highly desirable to capture real-time biological information from a "free" roaming laboratory animal housed in its home cage as depicted in Fig. 1.

Developing such a system requires studying sensing methods for various vital signals and low-power electronics design. Among all the biological signals, blood pressure is one of the most important vital signals, and there is no good solution for its longterm in vivo monitoring to date. The most common techniques used in small laboratory animal monitoring rely on an invasive catheter-tip transducer inserted into an artery or a tail cuff device. The catheter-tip transducers require a complex surgical procedure and can potentially suffer from an increased blood pressure, blood clotting, and reduced sensitivity with drift over time. Furthermore, discrete electronics are typically employed to implement the system, thus resulting in a large form factor with an excessive power dissipation, which in turn calls for a bulky ferrite-based RF coil for external RF powering or battery recharging. A final system typically exhibits a weight of approximately 10 $-20 \%$ of an animal body weight, thus severely limiting the accuracy of the measured biological signals due to post-implant trauma. Tail cuffs are inadequate for long-term monitoring because they constrain animal movement, which causes stress-induced signal distortion. Furthermore, tail cuffs can only obtain systolic and diastolic blood pressure levels instead of a continuous blood pressure waveform, which is highly desirable for

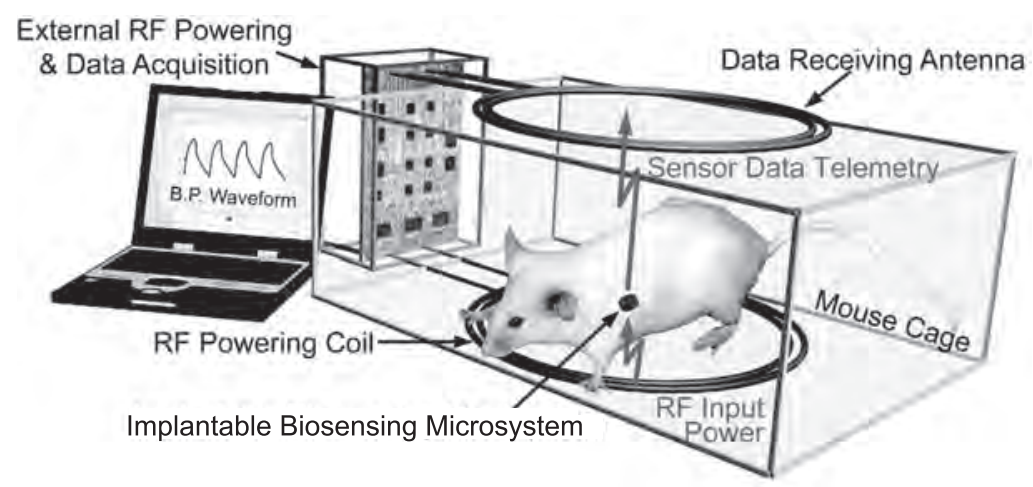

Fig. 1. In vivo real-time wireless biological signal monitoring system. 
biomedical and system biology research. Miniature implantable pressure sensor cuffs for tonometric blood pressure measurement have been proposed.(2) The technique can solve the above-mentioned issues associated with the conventional methods but significantly deforms the blood vessel shape, which can cause adverse physiological effects to the vessel property and thus may not be suitable for long-term monitoring. It is, therefore, highly desirable to develop a long-term implantable blood pressure monitoring system without the aforementioned concerns.

\section{Wireless Implantable Blood Pressure Monitoring}

A proposed wireless implantable blood pressure monitoring system is shown in Fig. 2. The system employs an instrumented elastic circular cuff, wrapped around a blood vessel to sense real-time blood pressure waveforms. The elastic circular cuff is made of biocompatible elastomer and is filled with low viscosity biocompatible insulating fluid, for example silicone oil, with an immersed MEMS pressure sensor and integrated electronic system. The MEMS sensor measures the pressure waveform in the cuff coupled from the expansion and contraction of the vessel. The measured waveform represents a scaled version of the vessel blood pressure waveform and can be processed by a nearby low-power integrated electronics, consisting of MEMS sensor interface circuitry, analog-to-digital converter (ADC) and system configuration and control unit, for signal conditioning and coding followed by wireless data telemetry to an external receiver. Figure 3 shows the overall electronic system architecture, where an adaptive RF-DC power converter is incorporated to provide sufficient and stable energy to the system implanted in an untethered animal.(3) RF powering has been widely used for biomedical implants, where both transmitting and receiving units are properly placed at a fixed distance from each other with a constant RF power coupling coefficient. However, in this research the receiving unit is implanted inside a freely moving laboratory animal resulting in a continuously changing RF power coupling. Therefore, a remote RF powering system with an adaptive power control capability is required to provide a
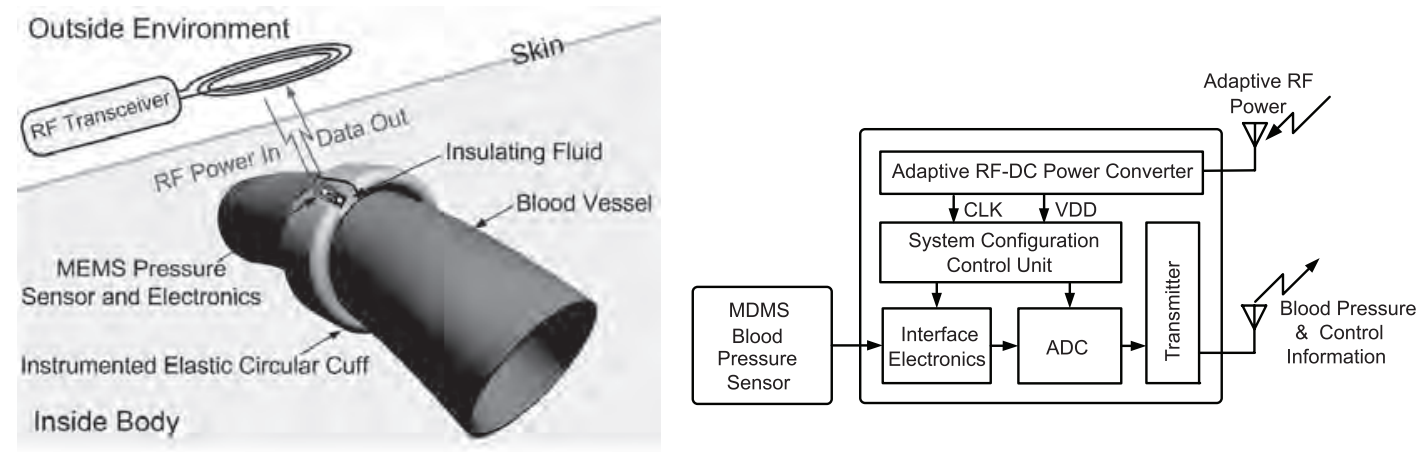

Fig. 2 (left). Wireless less-invasive implantable blood pressure monitoring.

Fig. 3 (right). Implant electronic system architecture. 
sufficient and stable energy to power the implantable microsystem in a varying magnetic coupling field. In addition, RF powering is used to eliminate the need for a battery, thus substantially reducing the overall implant size and weight. Furthermore, a miniature RF powering coil can be used owing to the low power budget of the integrated electronics, thus minimizing the total system size and weight.

The proposed design avoids vessel occlusion, bleeding, and blood clotting associated with the conventional catheter-tip-based technique. Furthermore, since the cuff is made of soft elastic material, the restrictive effect on the vessel is substantially minimized, and thus suitable for long-term monitoring. The sensor, electronics, and RF powering coil are inside the cuff as depicted in Fig. 2 for illustration purposes. In the final system design, the coil will be located outside the cuff to minimize packaging complexity.

\section{Implantable Blood Pressure Measurement Cuff}

Figure 4 shows a detailed 3-D configuration of the implantable blood pressure monitoring cuff, where a rigid silicone isolation ring is used to decouple the sensing cuff, which is located at the structural center, from environmental variations in animal body. Because of the soft nature of the sensing cuff outside wall, the pressure inside the sensing cuff is susceptible to environmental variations, such as animal muscle and tissue movement without the isolation ring. The sensing cuff outside wall can be made more rigid to decrease the effect; however, at the same time, the restraint to the blood vessel will increase, thus leading to a trade-off between blood pressure baseline drift and the vessel constraint. However, it is difficult to quantify this trade-off due to limited research data currently available on the long-term influence of laboratory animals with different amounts of vessel constraint. Therefore, a rigid isolation ring is employed to suppress the low-frequency drift. ${ }^{(4)}$

The isolation ring is designed so that an air cavity between the isolation ring and the sensing cuff outside wall is formed upon completion of the fabrication process. As a result, the sensing cuff outside wall can move freely in response to the blood vessel

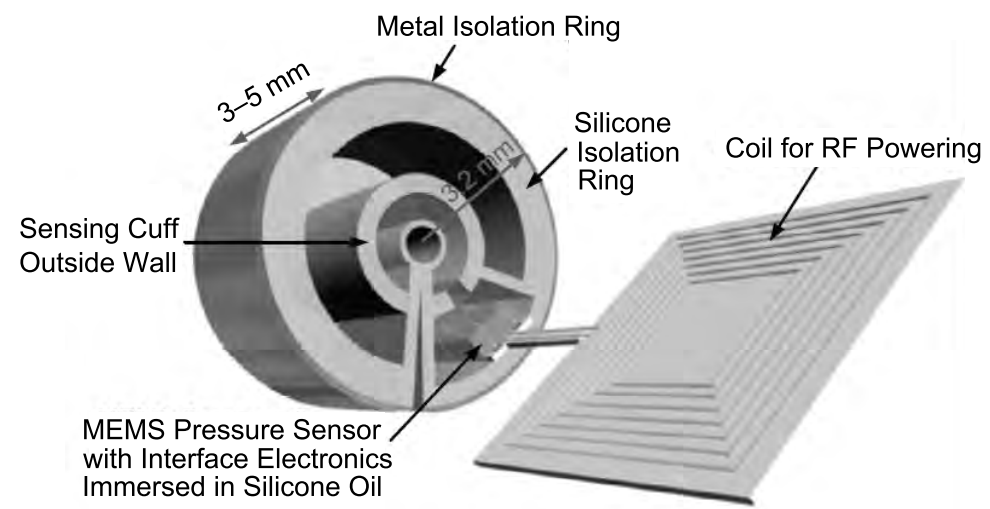

Fig. 4. Three-dimensional configuration of the blood pressure measurement cuff. 
pressure waveform. The wall thickness of the isolation ring is designed to be $1.5 \mathrm{~mm}$ to ensure a proper rigidity for an adequate isolation. In the figure, an additional thin metal layer is illustrated to wrap around the isolate ring for further suppression of environment variations. The overall structure exhibits a radius of $3.2 \mathrm{~mm}$ and a width of $3-5 \mathrm{~mm}$ to establish an adequate contact area with a blood vessel to be inserted in the middle of the cuff. The radius of the sensing cuff inside wall is designed to be approximately $0.5 \mathrm{~mm}$ with an outside sensing cuff wall radius of approximately $0.9 \mathrm{~mm}$, matching a typical dimension of small laboratory animal artery. The MEMS pressure sensor and integrated electronics are immersed in silicone oil and are enclosed inside the housing cavity exhibiting a dimension of approximately $2 \mathrm{~mm} \times 2 \mathrm{~mm} \times 2.5-4.5 \mathrm{~mm}$ as shown in Fig. 4. A spiral coil for RF powering is located outside the cuff with sealed feed-through connections to the electronics.

The silicone cuff is fabricated by conventional molding and bonding techniques. ${ }^{(5)}$ To ease implant procedures, it is desirable to obtain a precurved internal sensing cuff structure with a circular profile, which can be readily wrapped around a blood vessel during implantation and obtain a uniform contact to the vessel side wall to achieve a reliable measurement. The internal cuff is fabricated by attaching a silicone base with a predefined geometry to a prestretched thin silicone membrane using the fabrication process shown in Fig. 5. Owing to the built-in membrane stress, the attached layers would produce a desired circular profile upon fabrication completion. Biomedical-grade

(a)

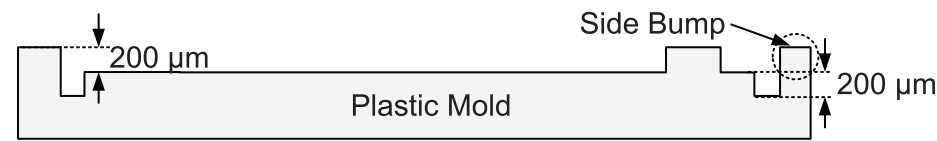

(b)

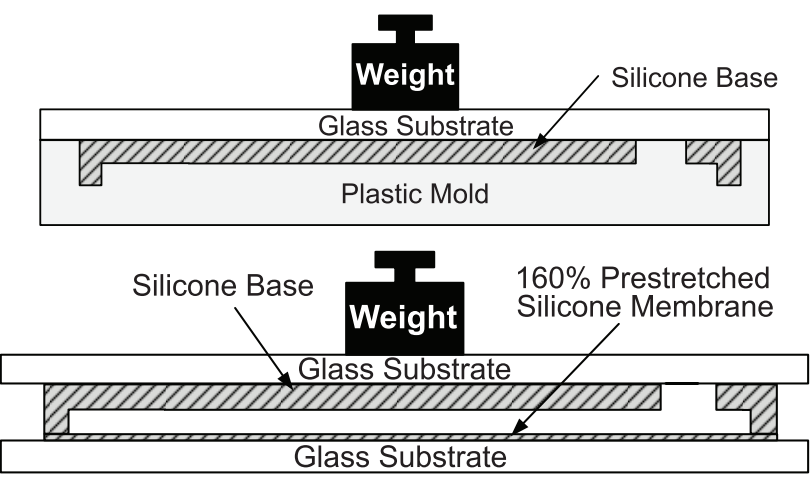

(d)

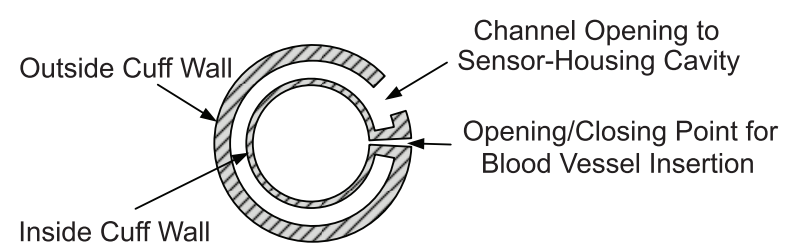

Fig. 5. Internal monitoring cuff fabrication process. 
silicone (MDX4-4210) from Dow Corning Company is selected to implement the cuff because of its biocompatibility and soft elastic properties. The process begins by making a plastic mold through a standard machining technique for constructing the silicone base with the cross-sectional view of the mold shown in Fig. 5(a). The elastomer material is then poured onto the mold surface, followed by a de-gassing step, which results in an air-free silicone uniformly covering the mold surface. Next, a thin glass substrate is positioned over the elastomer material with an applied weight on top. Together with the side bumps of $200 \mu \mathrm{m}$ height, a proper base thickness can be ensured as shown in Fig. 5(b), followed by curing at $40^{\circ} \mathrm{C}$ for $5 \mathrm{~h}$. The base exhibits a length of $5 \mathrm{~mm}$, a width of $3 \mathrm{~mm}$, and a thickness of $200 \mu \mathrm{m}$, which is adequate for a reliable wrapping around a blood vessel of a small laboratory animal. At this point, a thin commercial silicone membrane sheet of $40 \mu \mathrm{m}$ thickness is stretched to approximately $160 \%$ of its original length along the surface of another glass substrate to ensure its flatness. The $160 \%$ stretching is determined to be sufficient for obtaining a desired cuff profile upon the completion of the fabrication process. The membrane is then bonded to the silicone base side wall by applying a thin biocompatible adhesive layer with external force, followed by a curing step to form the cuff as shown in Fig. 5(c). The cuff is then peeled off from the two glass substrates. Owing to the built-in membrane prestretching stress, the structure curves into the desired profile as shown in Fig. 5(d).

The silicone isolation ring is fabricated by bonding two silicone parts (Part A and Part B) together with the fabrication process shown in Fig. 6 . The process also begins by making plastic molds through a standard machining technique. Biomedical-grade Class VI stiff elastomer material (C6-570) from Dow Corning Company is then poured onto the mold

(a)
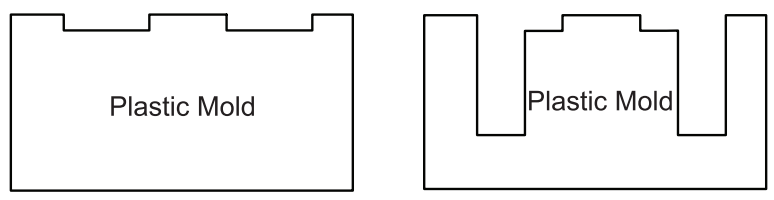

(b)

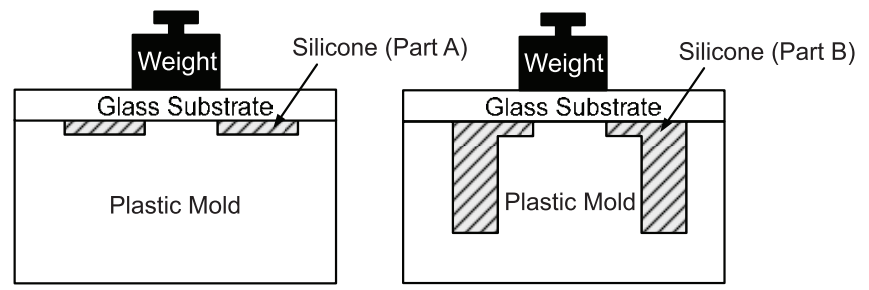

(c)
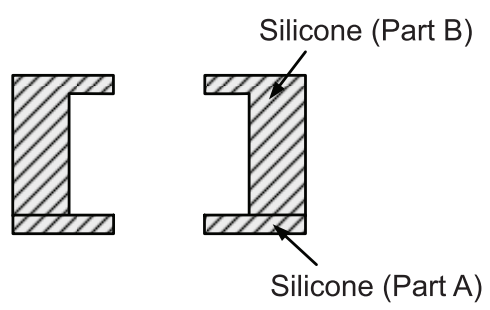

Fig. 6. Isolation ring fabrication process: (a) mold for Part A and mold for Part B, (b) molding for Part A and molding for Part B, (c) formed isolation ring. 
surface, followed by a degassing step. The stiff elastomer is chosen for isolation ring rigidity. Next, a thin glass substrate is positioned over the elastomer material with an applied weight on top to ensure a proper thickness as shown in Fig. 6(b), followed by curing at $110^{\circ} \mathrm{C}$. After curing, Parts $\mathrm{A}$ and $\mathrm{B}$ are peeled off from the molds and bonded together by applying a thin layer of biocompatible adhesive film and external force, followed by a curing step to form the isolation ring as shown in Fig. 6(c). The isolation ring is then carefully glued to the internal cuff outside wall using biocompatible silicone adhesive to complete the monitoring cuff, as shown in a 3D illustration in Fig. 7.

Figure 8 shows a picture of a fabricated cuff. Suture thread in the picture is used to secure the cuff in position with a blood vessel during an implant. With a commercial piezoresistive pressure sensor of $2.5 \mathrm{~mm} \times 3.5 \mathrm{~mm} \times 1.2 \mathrm{~mm}$ size used for the prototype implant, a rigid tubing is required to house the device. Such tubing will be eliminated in the final system design with miniature MEMS sensor and electronics. The cuff has a weight of $360 \mathrm{mg}$ excluding the rigid tubing.

Laboratory rats are chosen as a vehicle for cuff implant feasibility study. The cuff is wrapped around the right carotid artery for blood pressure monitoring and a commercial catheter-tip transducer is inserted into the left carotid artery as a reference for comparison, as shown in Fig. 9; note that a thin metal layer is wrapped around the silicone isolation ring for further isolation and protection.

Figure 10 shows a measured waveform from the monitoring cuff with a reference waveform from a catheter-tip transducer over $0.9 \mathrm{~s}$, indicating a scaling factor of approximately 0.25 with a heart rate of $250 \mathrm{bpm}$. Measurement over an extended time frame reveals a low-frequency drift of approximately $1 \mathrm{mmHg}$. Further investigation confirms that the low-frequency drift is caused by the piezoresistive pressure sensor due to ambient temperature fluctuation. With the functional monitoring cuff, a miniaturized pressure sensor of submillimeter size and low-power integrated electronics are required to realize the proposed miniature biosensing implant microsystem.
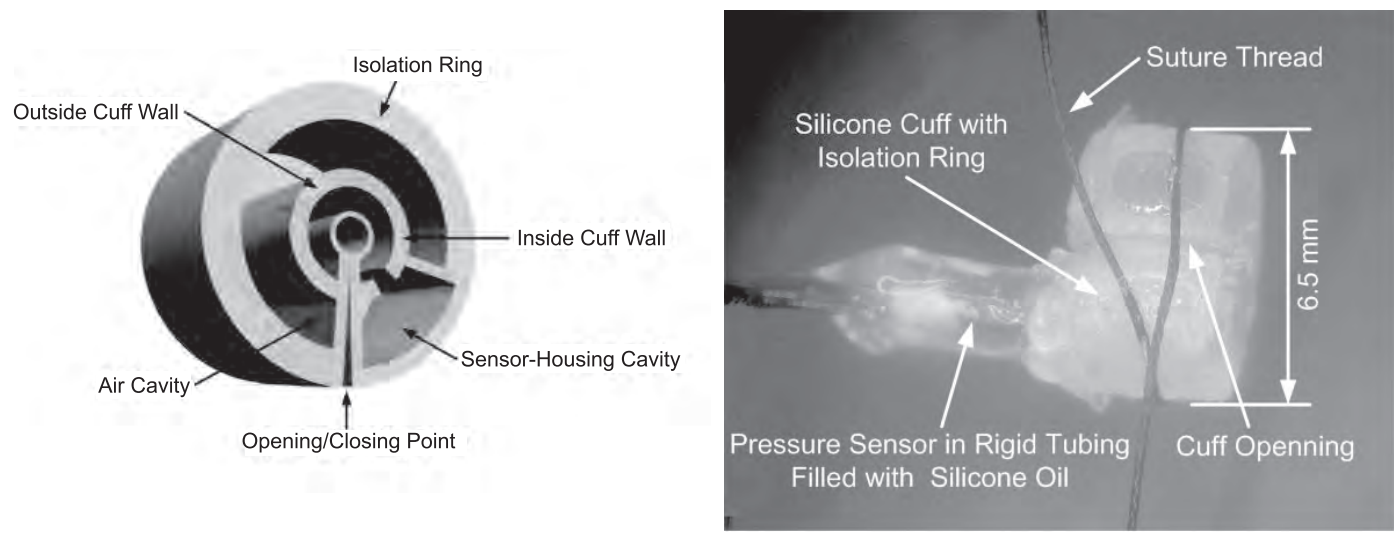

Fig. 7 (left). Three-dimensional illustration of fabricated monitoring cuff. Fig. 8 (right). Fabricated blood pressure monitoring cuff. 

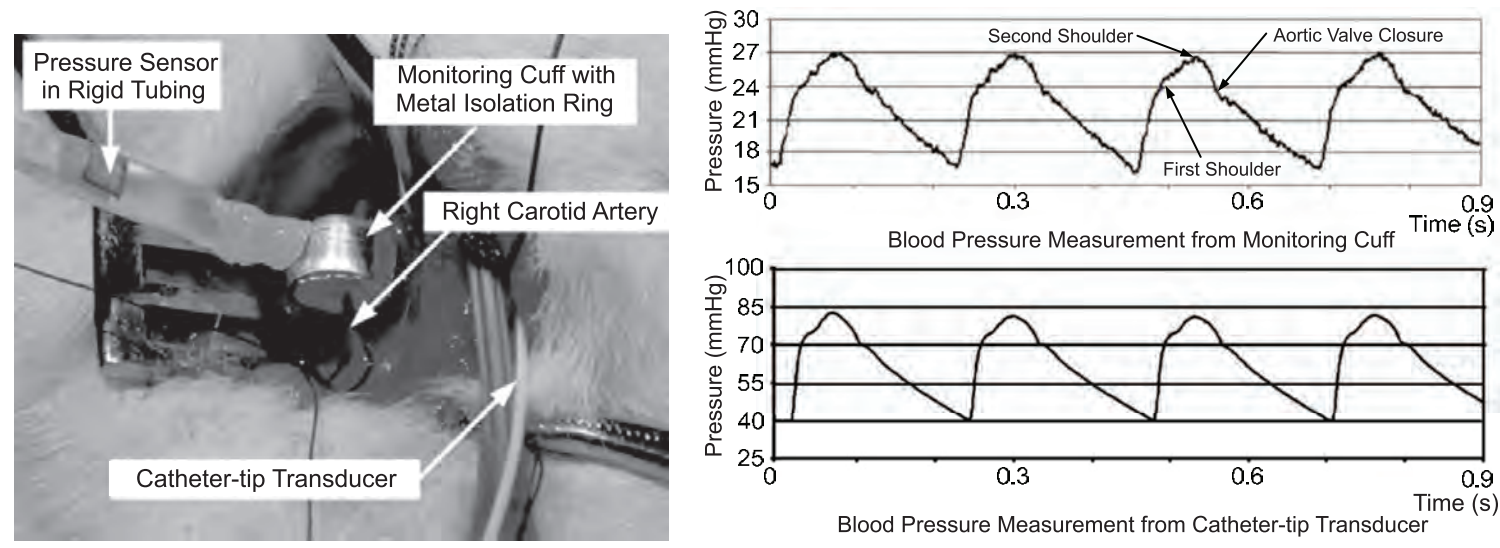

Fig. 9 (left). Laboratory rat implant trial.

Fig. 10 (right). Detailed blood pressure measurement waveforms.

\section{MEMS Capacitive Pressure Sensor}

MEMS capacitive pressure sensor is chosen for the proposed biomedical implant application owing to its miniature size, high sensitivity, low temperature dependence, zero DC power dissipation, and time stability. Figure 11 shows a cross-sectional view of a sensor, which consists of an edge-clamped silicon diaphragm over a vacuum cavity. The diaphragm deflects downward under an increased external pressure; hence, the increased device capacitance value. The sensor can be designed to operate in touch mode to obtain a nearly linear performance. ${ }^{(6)}$

An MEMS capacitive pressure sensor is designed and fabricated using a similar wafer bonding and etch-back process as described in Ref. 7. The fabrication process flow is presented in Fig. 12. First, a 4-inch P-type silicon wafer with a resistivity of 0.01 $\Omega \cdot \mathrm{cm}$ and $1.5 \mu \mathrm{m}$ oxide layers is patterned and wet-etched using standard oxide etchant to define the pressure sensor cavity as shown in Fig. 12(a), followed by a $1000 \AA$ dry oxidation on the silicon wafer surface as a dielectric layer shown in Fig. 12(b). An SOI wafer with a $2-\mu \mathrm{m}$-thick P-type $0.01 \Omega \cdot \mathrm{cm}$ structural layer is then bonded on the silicon wafer at room temperature followed by an annealing process at $1050^{\circ} \mathrm{C}$ to ensure a reliable bonding quality, as shown in Fig. 12(c). The structural layer is to be used as the pressure sensor diaphragm. Next, the backside of the silicon wafer is protected by black wax. The handle substrate of the SOI wafer is then removed in $40 \% \mathrm{KOH}$ solution at $55^{\circ} \mathrm{C}$. The $55^{\circ} \mathrm{C}$ is chosen as a trade-off between black wax protection capability and silicon etching rate, as the etching rate increases with temperature while wax is softened at elevated temperature. After dissolving the wax, the $2 \mu \mathrm{m}$ buried thermal oxide layer in the SOI wafer and the $1.5 \mu \mathrm{m}$ oxide layer on the back of the silicon wafer are removed using standard oxide enchant, as illustrated in Fig. 12(d). The structural layer is then patterned and silicon RIE is performed. The exposed oxide layer is then wet-etched to 


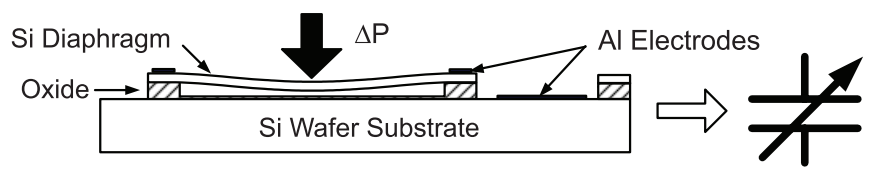

Fig. 11. Capacitive pressure sensor cross-sectional view.

(a)

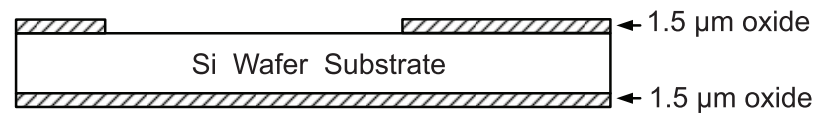

(b)

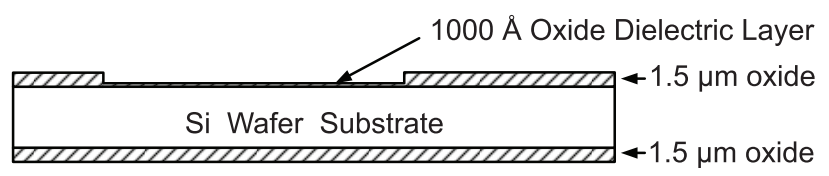

(c)

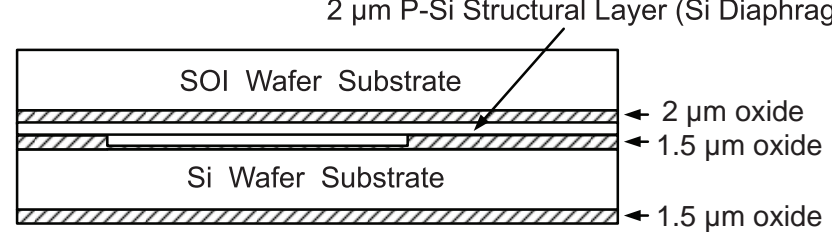

(d)

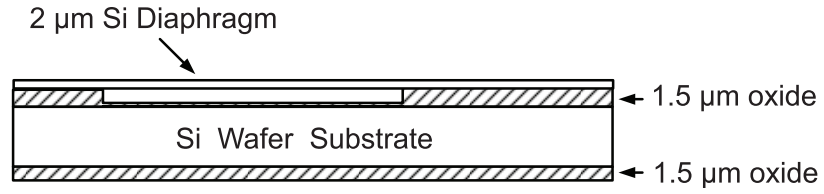

(e)
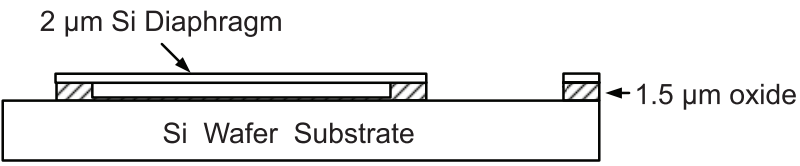

(f)

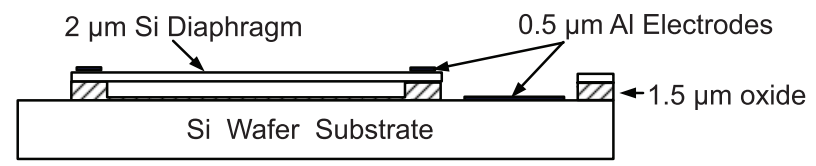

Fig. 12. MEMS capacitive pressure sensor fabrication process.

access the silicon substrate for electrical contact as shown in Fig. 12(e). Finally, the device is metalized by sputtering a $0.5-\mu \mathrm{m}$-thick aluminum layer, followed by patterning to form electrodes as shown in Fig. 12(f). 
Figure 13 shows SEM images of a fabricated pressure sensor exhibiting a dimension of $0.4 \mathrm{~mm} \times 0.5 \mathrm{~mm} \times 0.4 \mathrm{~mm}$ with a measured nominal capacitance value of $2 \mathrm{pF}$ and a sensitivity of approximately $0.7 \mathrm{fF} / \mathrm{mmHg}$. The device miniature size is adequate for enclosure in the blood pressure sensing cuff as depicted in Fig. 4.

\section{Implant Electronic System}

A batteryless low-power implant electronic system is critical for realizing the proposed wireless microsystem. Figure 14 shows the overall electronic system design.

An external RF power signal is coupled into the implant electronics via a tuned LC network, followed by a CMOS voltage doubler to produce two DC voltages, $V_{\text {HIGH }}$ and $V_{\text {Low }}$, which are further regulated to generate a stable $3 \mathrm{~V}$ supply for controlling MOSFET switches and $2 \mathrm{~V}$ supply for low-power electronics; note that two separate $2 \mathrm{~V}$ supplies are produced for analog and digital electronics in order to minimize digital switching noise coupling. Owing to the dynamic movement of a freely moving mouse, the received RF power can vary drastically as a function of mouse position and tilting
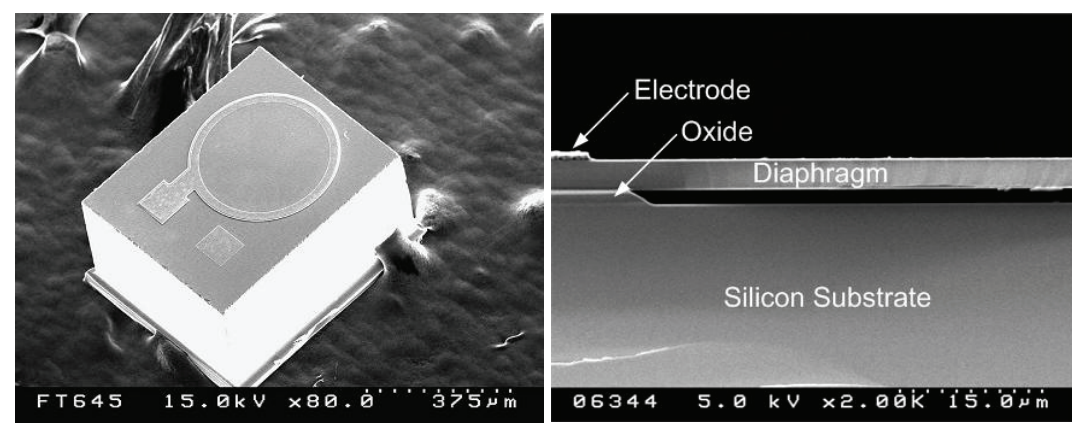

Fig. 13. SEMs of fabricated MEMS capacitive pressure sensor: (a) 3-D view, (b) cross sectional view.

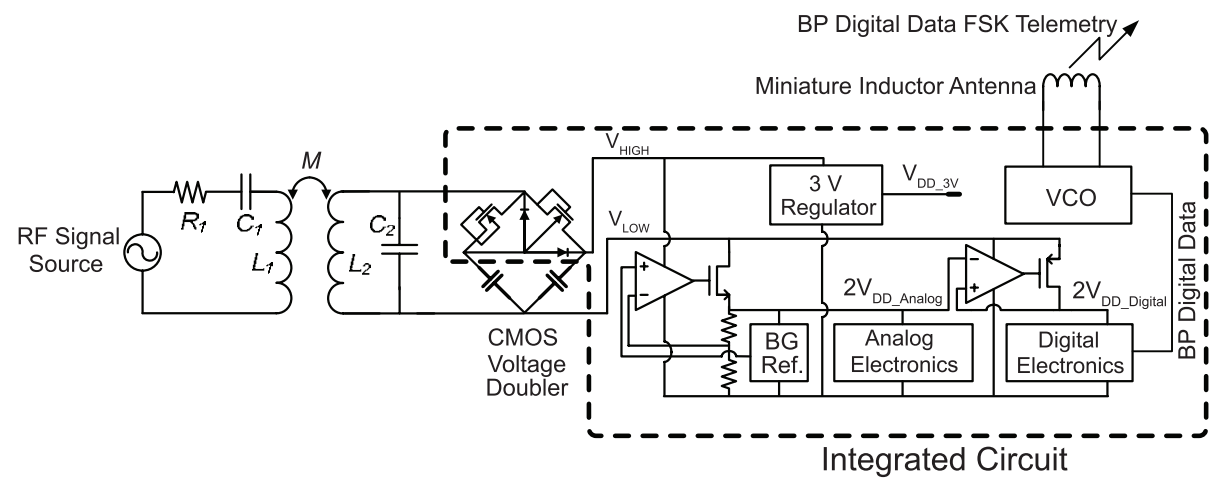

Fig. 14. Overall implant electronic system design. 
angle with respect to the external RF coil.(8) Therefore, an integrated power sensor is incorporated in the design (not shown in the figure for simplicity) to sense the incoming power level and feedback a 1-bit control signal to the external RF power source to adaptively adjust the transmitted power. ${ }^{(3)}$ This technique not only regulates the internal voltage swing in implant electronics, which is critical to ensure proper device operation and stable DC supplies, but also minimizes the external power dissipation; hence, environment heating as well as tissue absorption of RF power. The digitized blood pressure (BP) signal is transmitted along with the 1-bit power control signal to an external receiver nearby by a $433 \mathrm{MHz}$ VCO transmitter through frequency-shift keying (FSK) modulation scheme. A discrete miniature surface-mount inductor is used to implement the VCO tank as well as the transmitting antenna owing to its high $Q$ value, thus minimizing transmitter current dissipation at $120 \mu \mathrm{A}$. Active telemetry scheme is desirable owing to its reliable signal transmission independent of implant position and orientation. A low-power switched-capacitor fully differential capacitance-to-voltage (C/ $\mathrm{V})$ converter is designed to interface with the MEMS capacitive pressure sensor, which achieves a resolution of approximately $100 \mathrm{aF}$ over $1 \mathrm{kHz}$ bandwidth, corresponding to a $0.1 \mathrm{mmHg}$ pressure change exerting on the MEMS pressure sensor. With a typical scaling factor of 0.1 , a blood vessel pressure change sensing resolution of $1 \mathrm{mmHg}$ can be expected. Furthermore, an automatic capacitance offset cancellation scheme is designed to generate a closely matched reference capacitor for the MEMS sensor, as shown in Fig. 15. During the initial phase of the circuit operation, a digitally controlled capacitor reference array at the amplifier input will be cycled through to find a reference capacitance closely matching the sensor nominal capacitance. The digital reference capacitor can greatly suppress the output offset voltage, thus ensuring a proper circuit operation and dynamic range requirement. The $\mathrm{C} / \mathrm{V}$ converter output is then digitized by an 11-bit cyclic ADC, followed by Manchester coding and FSK data telemetry. Figure 16 shows a micrograph of an IC implementing all the required electronic functions.

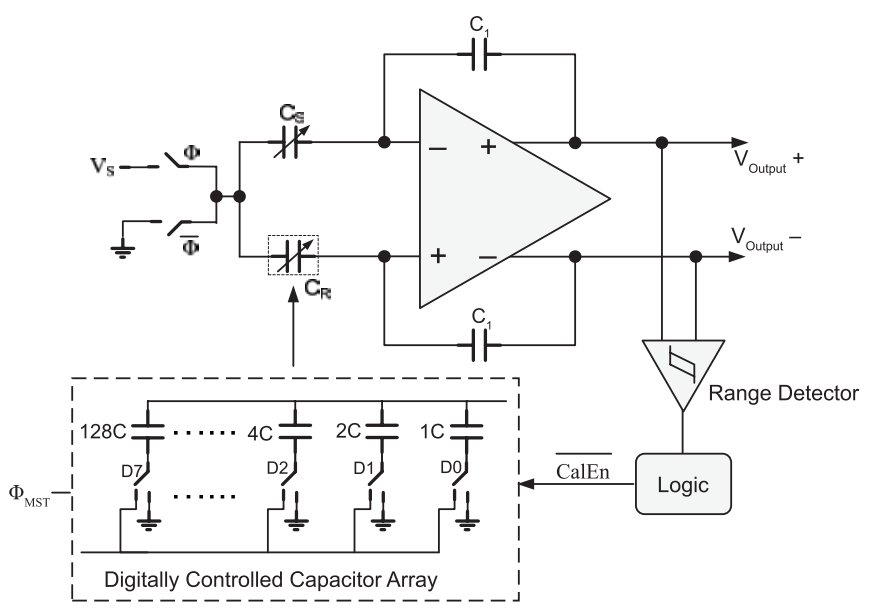

Fig. 15. C/V converter with automatic capacitance offset cancellation. 
The chip occupies an area of $2 \mathrm{~mm} \times 2 \mathrm{~mm}$ and consumes $150 \mu \mathrm{A}$ DC current from $2 \mathrm{~V}$ supply. The measured regulators' DC output voltages at $2 \mathrm{~V}$ and $3 \mathrm{~V}$ are $2.1 \mathrm{~V}$ and 3.2 $\mathrm{V}$, respectively, when a $4 \mathrm{MHz}$ power signal with an equivalent amplitude of $2.9 \mathrm{~V}$ is applied to the LC tank. FSK power spectrum measurement reveals a $300 \mathrm{kHz}$ frequency spacing, which is designed to accommodate the required data rate. Figure 17 shows the transmitted and received Manchester-coded testing data with a $15 \mathrm{~cm}$ separation achieving a worst case bit-error rate (BER) of $10^{-3}$, limited by the commercial receiver performance.

\section{6. $\quad$ System Testing and Packing}

The MEMS pressure sensor is then directly bonded over the IC by epoxy and wire bonded to form electrical connections for the prototype system. The module is then interfaced with RF powering coil exhibiting 20 turns and $5 \mathrm{~mm}$ in diameter, and discrete components over a thin flexible substrate. Four miniature surface-mount components are employed in the prototype design: three capacitors (two for CMOS voltage doubler rectifier and one for internal coil tank) and one FSK VCO inductor, as shown in Fig. 18. These components can be potentially integrated on chip at the expense of silicon area and power dissipation. The unit is tested inside a metal pressure chamber for system characterization. In this testing, the metal pressure chamber will block the RF signal; therefore, RF signal with a frequency of $4 \mathrm{MHz}$ and an amplitude of $2.9 \mathrm{~V}$ is generated by an external function generator and coupled to the LC tank connected to the microsystem through chamber feed-through wires to mimic the RF powering. The RF signal is then rectified and regulated on chip to power the microsystem. The Manchestercoded data is also recorded by a data requisition system through chamber feed-through
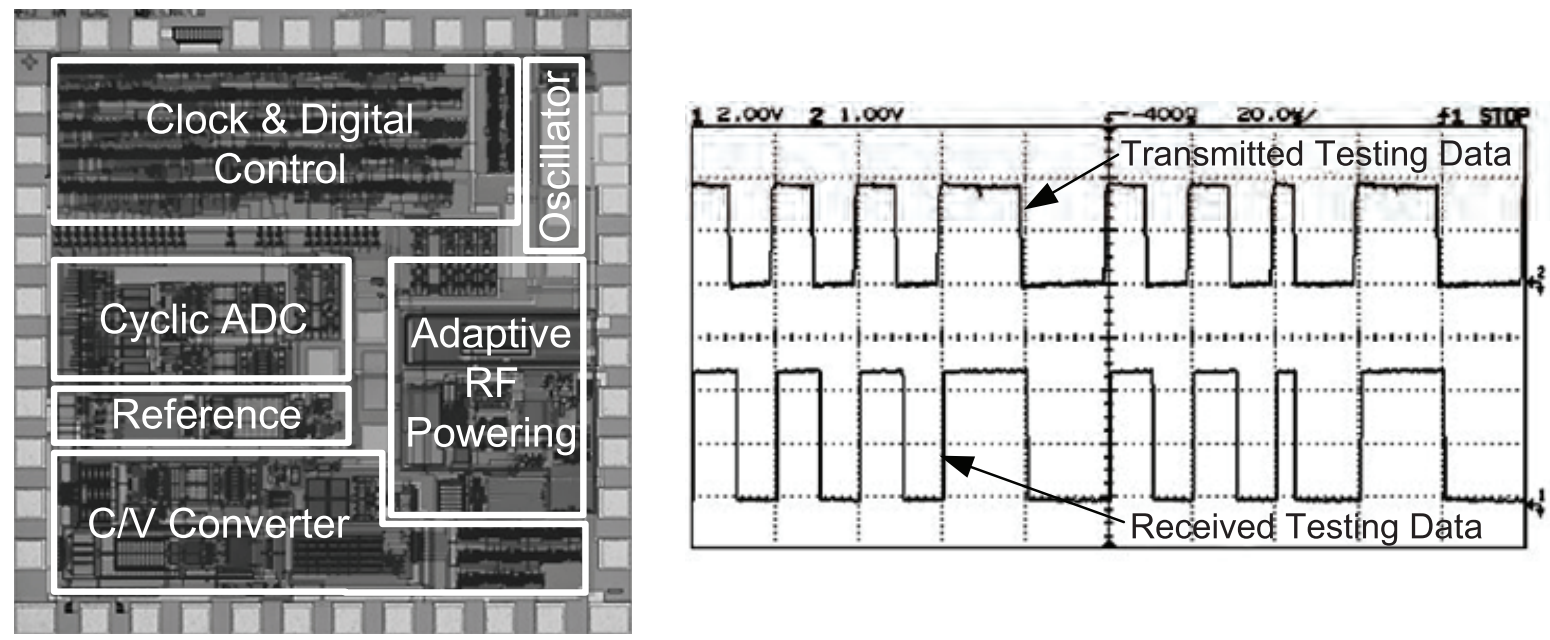

Fig. 16 (left). Micrograph of implant electronic system design.

Fig. 17 (right). Transmitted and received testing data. 


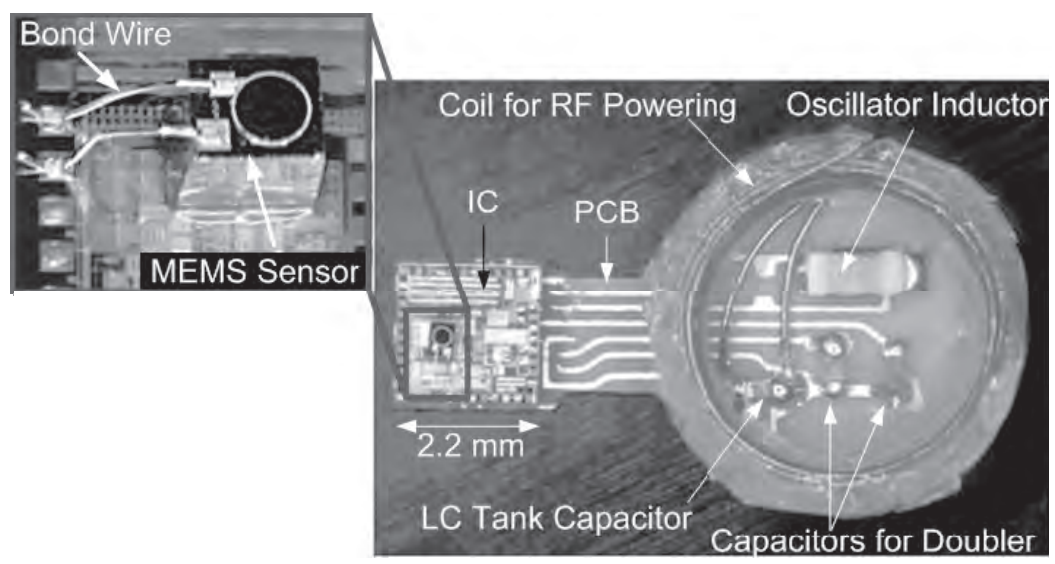

Fig. 18. Electronic system packaging.
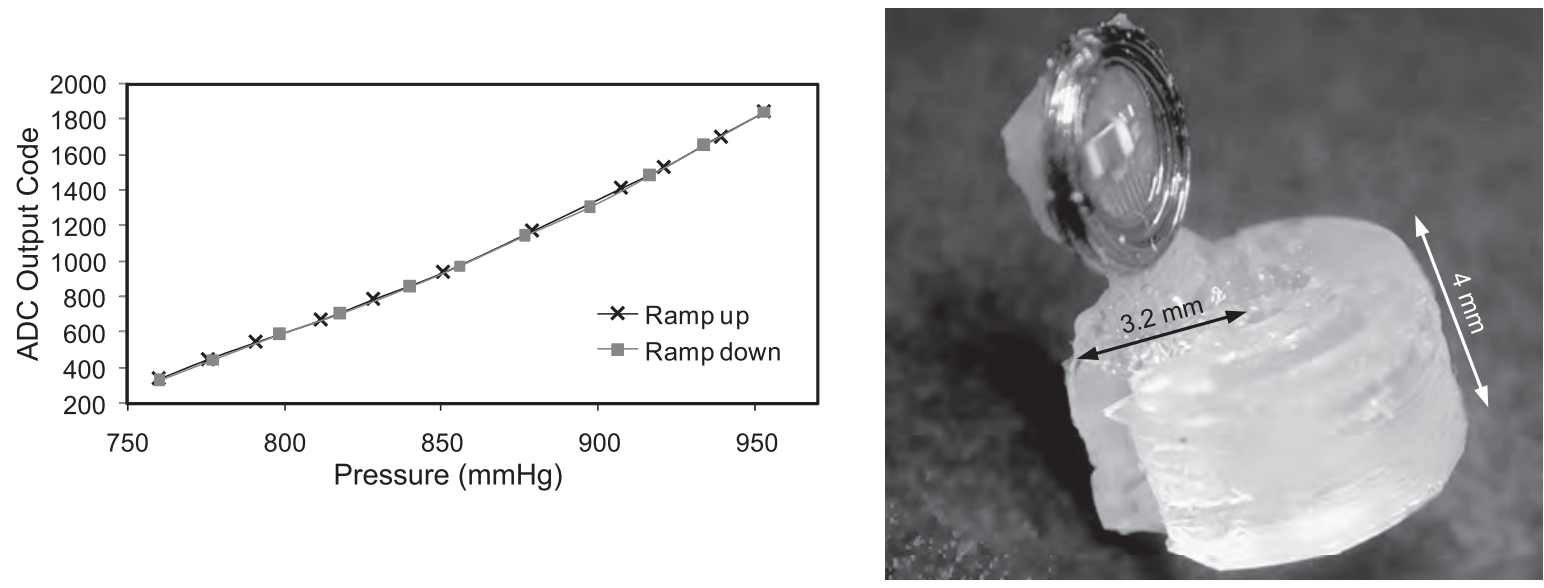

Fig. 19 (left). ADC digital output code versus pressure variation.

Fig. 20 (right). Packaged blood pressure monitoring cuff.

wires. The recorded data is then processed in Matlab to obtain the digital representation of input pressure information. Figure 19 shows the measured output digital code versus a $200 \mathrm{mmHg}$ pressure variation, indicating an average system sensitivity of $7 \mathrm{LSB} / \mathrm{mmHg}$ with a hysteresis below $1 \%$ of full scale and nonlinearity below $2.5 \%$ of full scale. The nonlinearity is due to the MEMS capacitive pressure sensor operating in nontouch mode, which is also responsible for the low hysteresis. The total noise is measured to be 800 $\mu V_{\text {RMS }}$ referred to the ADC input, which is equivalent to $0.1 \mathrm{mmHg}$ resolution with $59 \mathrm{~dB}$ dynamic range. This achieved performance is adequate for the microsystem to obtain reliable and accurate blood pressure information.

The overall microsystem is then coated with a thin silicone layer for system protection. Experimental characterization shows that the MEMS capacitive pressure 
sensor performance is insensitive to the silicone coating. The overall electronic system is then inserted in a blood pressure monitoring cuff with a silicone feed-through seal as shown in Fig. 20. The cuff exhibits a diameter of $6.5 \mathrm{~mm}$, a length of $4 \mathrm{~mm}$, and a total weight of $330 \mathrm{mg}$, representing one order of magnitude mass reduction compared with existing commercial sensing technology. The prototype blood pressure monitoring cuff is expected with obtain acute and long-term small laboratory animal real-time blood pressure signals.

\section{Conclusion}

The design and development of a novel wireless implantable blood pressure sensing microsystem is presented. Realizing such a system requires conventional molding and machining process with a minimum feature size of sub-mm, MEMS micromachining technology with micrometer $(\mu \mathrm{m})$-scale resolution, and sub- $\mu \mathrm{m}$ low power CMOS integrated circuit technology. The proposed microsystem is expected to make a significant impact on biomedical and system biology research.

\section{Acknowlegement}

This work is supported by the National Science Foundation under grant \# EIA-0329811 and Case Prime Fellowship from Case School of Engineering at Case Western Reserve University.

\section{References}

1 B. Hoit, S. Kiatchoosakun, J. Restivo, D. Kirkpatrick, K. Olszens, H. Shao, Y. Pao and J. Nadeau: Genomics 79 (2002) 679.

2 B. Ziaie, T. Wu, N. Kocaman, K. Najafi and D. J. Anderson: IEEE Solid-State Sensor and Actuator Workshop, June 1998 (South Carolina, 1998) p. 216.

3 N. Chaimanonart, M. D. Zimmerman and D. J. Young: 7th IEEE Conference on Sensors, October 2008 (Lecce, 2008).

4 P. Cong, D. J. Young, B. Hoit and W. H. Ko: 28th Annual International Conference of the IEEE Engineering in Medicine and Biology Society (EMBS ‘06) (New York, 2006) p. 1854.

5 P. Cong, K. Olszens, D. J. Young and W. H. Ko: 13th International Conference on Solid-State Sensors, Actuators and Microsystems, June 2005 (Seoul, 2005) p. 2002.

6 W. H. Ko and Q. Wang: Sens. Actuators, A 75 (1999) 242.

7 D. J. Young, J. Du, C. A. Zorman and W. H. Ko: IEEE Sensors Journal, Special Issue on Microsensors and Microactuators, August 2004, p. 464.

8 M. Zimmerman, N. Chaimanonart and D. J. Young: 28th Annual International Conference of the IEEE Engineering in Medicine and Biology Society (EMBS’06) (New York, 2006) p. 2506.

9 P. Cong, W. H. Ko and D. J. Young: Custom Integrated Circuits Conference (CICC 2008), September 2008 (San Jose, 2008) p. 523. 